\title{
Transformation Thermodynamics with Arbitrarily Shaped Non-Conformal Objects and Coatings
}

\author{
Qingxiang $\mathrm{Ji}^{1}$, Guodong Fang ${ }^{1}$ and Jun Liang ${ }^{2}$ \\ ${ }^{1}$ Center for Composite Materials, Harbin Institute of Technology, Harbin, 150001, China \\ ${ }^{2}$ Institute of Advanced Structure Technology, Beijing Institute of Technology, Beijing, 100081, China
}

\begin{abstract}
In this paper we apply transformation optics theory to thermodynamics and design thermal cloaks and concentrators with arbitrarily shaped non-conformal objects and coatings. Expressions of the required material parameters are derived analytically and then validated by numerical simulations. We apply this method to design a thermal cloak which can guide the heat flow around the inner domain without perturbation to external thermal fields. In this way, the object inside the inner domain is protected from the invasion of external heat fluxes. In contrast, a concentrator is designed to concentrate heat flows into a small region without disturbing outside temperature fields, which can considerably enhance the heat density in the designed domain. The proposed method extends the design flexibility in manipulating heat flux and will find wide applications in thermal protection systems, solar cells and so on.
\end{abstract}

\section{Introduction}

Transformation optics (TO) theory was first proposed by Pendry [1] and Leonhardt [2] in the field of achieving invisibility of electromagnetic waves. Since then TO attracts extensive interests and leads to considerable achievements in theoretical and experimental approaches extended to many fields, such as acoustics [3-5], magnetic field [6-8], elastic wave $[9,10]$ and matter waves $[11,12]$. TO theory was also applied to thermodynamics since the diffusion equation retains its form under coordinate transformation [13-16]. Thermal cloaks, concentrators and other devices are designed based on coordinate transformations and then experimentally realized $[17,18]$. These pioneer works theoretically demonstrate the possibilities for cloaking and focusing heat flux. Expressions of related diffusivity in a general coordinate system are also derived.

However, previous works are mainly focused on regular geometry such as cylindrical and spherical shapes. The geometric requirements impose great restrictions on practical applications and further research is needed. Guenneau [16] proposed two-dimensional thermal cloaks and concentrators with arbitrarily shaped objects and coatings. Yang [19] investigated a two-dimensional heat flux cloak with arbitrarily shaped objects and verified its theory by numeric results, but the work was about homotopy mapping that achieved same shaped objects and coatings.

In this paper, we propose three-dimensional heat flux devices with arbitrarily shaped non-conformal objects and coatings. General expressions of the transformation based parameters are derived. Then some finite element computations are performed, which demonstrates the designed properties of the cloaks and concentrators, consequently, verifies the validity of the proposed method. One of the practical strengths of this paper lies with its extension in design flexibility in manipulating heat fluxes.

\section{Methodology}

We consider a three dimensional diffusion problem without a heat source, of which the heat conduction equation can be written as

$$
\nabla \cdot(\sigma \nabla T)+c \rho \frac{\partial T}{\partial t}=0 .
$$

where $\sigma$ is the heat conductivity and $T$ is the temperature, $c$ and $\rho$ are the specific heat and material density, respectively.

Earlier works [10] in transformation thermodynamics has demonstrated that the conduction equation is invariant in its form under the coordinate transformation

$$
\nabla^{\prime} \cdot\left(\sigma^{\prime} \nabla^{\prime} T^{\prime}\right)+c^{\prime} \rho^{\prime} \frac{\partial T^{\prime}}{\partial t}=0
$$

when

$$
\boldsymbol{\sigma}^{\prime}=\frac{J \boldsymbol{\sigma} J^{T}}{\operatorname{det}(J)}, \rho^{\prime} c^{\prime}=\frac{\rho c}{\operatorname{det}(J)}
$$

In Eq. (3), the superscript denotes that the parameters are expressed in the transformed space. $\mathrm{J}$ is the Jacobian transformation matrix with its elements derived by

$$
J_{i j}=\left[\frac{\partial\left(x^{\prime}, y^{\prime}, z^{\prime}\right)}{\partial(x, y, z)}\right] \text {. }
$$

Then a linear geometric transformation is defined as 


$$
\left\{\begin{array}{l}
r^{\prime}=\alpha(\theta) r+\beta(\theta) \\
\theta^{\prime}=\theta \\
z^{\prime}=z
\end{array} .\right.
$$

Eq. (5) denotes a radial stretch or compression of polar coordinates that squeezes space from a volume into a shell surrounding the object domain. The parameters $\alpha(\theta)$ and $\beta(\theta)$ indicates that the stretch or compression scale depends on the azimuthal angle when modeling domains of a smooth arbitrary shape.

Then the thermal conductivity and capacity parameters in transformed space are derived by

$$
\sigma^{\prime}=\left[\begin{array}{ccc}
\sigma_{r} \cdot \frac{r^{\prime}-\beta}{r^{\prime}}+\sigma_{\theta} \cdot \frac{\lambda^{2}}{r^{\prime}\left(r^{\prime}-\beta\right)} & \sigma_{\theta} \cdot \frac{\lambda}{r^{\prime}-\beta} & 0 \\
\sigma_{\theta} \cdot \frac{\lambda}{r^{\prime}-\beta} & \sigma_{\theta} \cdot \frac{r^{\prime}}{r^{\prime}-\beta} & 0 \\
0 & 0 & \sigma_{z} \cdot \frac{r^{\prime}-\beta}{\alpha^{2} r^{\prime}}
\end{array}\right]
$$

where $\lambda=\partial r^{\prime} / \partial \theta$.

One should note that $\lambda$ is not a constant value and varies in different transformations, which will be discussed in details in following sections. Clearly, the derivation of $\lambda$ is the key step in cloaking and concentrating heat flux.

\section{Results and discussion}

\subsection{An invisibility cloak}

In this section we consider a two dimensional cloak based on the linear geometric transformation in Eq. (5). The inner boundary (boundary of the object) and outer boundary (boundary of the coating) are defined respectively by smooth closed curves $R_{1}(\theta)$ and $R_{2}(\theta)$ which depend upon the angular variable $\theta$. We have parameters in this section expressed as follows:

$$
\alpha=\frac{R_{2}(\theta)-R_{1}(\theta)}{R_{2}(\theta)}, \beta=R_{1}(\theta) .
$$

By Eq. (8), we will map the field within the object domain $r \leq R_{2}(\theta)$ onto the coating domain $R_{1}(\theta) \leq r^{\prime} \leq R_{2}(\theta)$. The region $r^{\prime} \leq R_{1}(\theta)$ defines the cloaking region while the coating area consists of a material with anisotropic conductivity given by

$$
\begin{aligned}
\lambda= & \frac{1}{R_{2}(\theta)\left(R_{2}(\theta)-R_{1}(\theta)\right)}\left\{\frac{d R_{1}(\theta)}{d \theta} R_{2}(\theta)\left(R_{2}(\theta)-r^{\prime}\right)\right. \\
& \left.-\frac{d R_{2}(\theta)}{d \theta} R_{1}(\theta)\left(R_{1}(\theta)-r^{\prime}\right)\right\}
\end{aligned}
$$

It is easy to verify that if the inner boundary and outer boundary of the cloak are circular, namely $d R_{1}(\theta) / d \theta=d R_{2}(\theta) / d \theta=0$, then $\lambda$ vanishes.

To demonstrate the validity of the designed cloak, some finite element computations with COMSOL
MULTIPHISICS are presented. We apply constant temperatures normalized to 1 and -1 respectively on the left side and right side while other sides are thermal insulated. The background material is defined as isotropic with unit conductivity. Different arbitrary geometries are used to describe the flexibility of the approach. Without loss of generality, the irregular boundaries of the cloak are defined on some different contour equations:

Case A:

$$
\begin{aligned}
& R_{1}(\theta)=0.6+0.1 \cos (\theta)+0.05 \sin (2 \theta)-0.1 \sin (3 \theta) ; \\
& R_{2}(\theta)=1.2+0.1 \sin (\theta)+0.2 \sin (3 \theta)
\end{aligned}
$$

Case B:

$$
R_{1}(\theta)=0.6 ; R_{2}(\theta)=1.2+0.1 \sin (\theta)+0.2 \sin (3 \theta)
$$

Case $\mathrm{C}$ :

$$
\begin{aligned}
& R_{1}(\theta)=0.6+0.1 \cos (\theta)+0.05 \sin (2 \theta)-0.1 \sin (3 \theta) \\
& R_{2}(\theta)=1.2
\end{aligned}
$$

Case D:

$R_{1}(\theta)=0.6 ; R_{2}(\theta)=1.2$

The simulated results for the temperature profile and the heat flux lines are shown in Fig. 1. The arrows display the pathway of the total heat flux while the white lines indicate the isothermal lines. It is seen that the heat flux pass around the object domain and eventually return undisturbed to their original trajectory as if they have passed through the object. Besides, the isothermal lines are smoothly bent around the inner region but perfectly excluded from these areas. In contrast, the temperature field inside the inner region is uniform and does not interact with the coating region. Therefore, the object inside the inner domain is protected from the invasion of the external heat flux.

It is seen that when the outer and inner boundaries are both circular the parameter $\lambda$ vanishes. In this way, a perfect cylindrical thermal cloak is proposed, as is shown in Fig. 1 (d). The designed cloak manipulates heat flux around the inner domain, consequently, leads to thermal protection of the hidden objects.

Thus, a thermal cloak for arbitrarily shaped objects and coatings is achieved. Traditional method of thermal protection, such as building in thermally insulated materials to block part of the heat flows, is too simple and has single function. Besides, previous cloaks focusing on symmetry and regular shapes are also limited in practical applications because of their strict geometric restrictions. The method proposed in this paper makes it possible to design a cloak with arbitrarily different shaped objects and coatings which goes beyond geometric constraints and leads to more practical and extensive applications. 


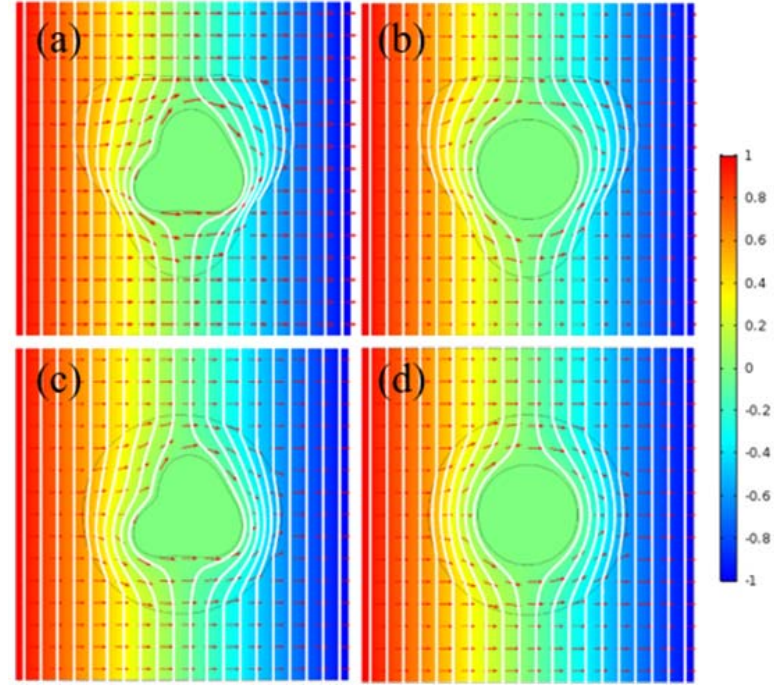

Fig. 1. Temperature field profiles of an invisibility cloak: (a) Case A; (b) Case B; (c) Case C; (d) Case D

\subsection{A thermal concentrator}

In this section we consider a two dimensional concentrator based on the linear geometric transformation in Eq. (5). The objects and coating area are defined by three embedded smooth closed curves $R_{1}(\theta)<R_{2}(\theta)<R_{3}(\theta)$, for $0 \leq \theta \leq 2 \pi$. We define $\alpha=\frac{R_{1}(\theta)}{R_{2}(\theta)}$ and $\beta=0$ for the domain $0<r<R_{2}(\theta)$, thus mapping the field in the region $0<r<R_{2}(\theta)$ onto the inner core $0<r^{\prime}<R_{1}\left(\theta^{\prime}\right)$. Similarly, by defining $\alpha=\frac{R_{3}(\theta)-R_{1}(\theta)}{R_{3}(\theta)-R_{2}(\theta)}$ and $\beta=R_{3}(\theta) \frac{R_{1}(\theta)-R_{2}(\theta)}{R_{3}(\theta)-R_{2}(\theta)}$ for the domain $R_{2}(\theta)<r<R_{3}(\theta)$, we can map the field in the region $R_{2}(\theta)<r<R_{3}(\theta)$ onto the coating area $R_{1}\left(\theta^{\prime}\right)<r^{\prime}<R_{3}\left(\theta^{\prime}\right)$. The resulting thermic parameters are divided into two parts:

for the inner core $0<r^{\prime}<R_{1}\left(\theta^{\prime}\right)$,

$$
\lambda=\frac{\frac{d R_{1}(\theta)}{d \theta} R_{2}(\theta)-\frac{d R_{2}(\theta)}{d \theta} R_{1}(\theta)}{R_{1}(\theta) R_{2}(\theta)} r^{\prime},
$$

for the coating area $R_{1}\left(\theta^{\prime}\right)<r^{\prime}<R_{3}\left(\theta^{\prime}\right)$,

$$
\begin{aligned}
& \lambda=-\frac{1}{\left(R_{3}(\theta)-R_{1}(\theta)\right)\left(R_{3}(\theta)-R_{2}(\theta)\right)} . \\
& \left\{\frac{d R_{1}(\theta)}{d \theta}\left(R_{3}(\theta)-R_{2}(\theta)\right)\left(R_{3}(\theta)-r^{\prime}\right)\right. \\
& -\frac{d R_{2}(\theta)}{d \theta}\left(R_{3}(\theta)-R_{1}(\theta)\right)\left(R_{3}(\theta)-r^{\prime}\right) \\
& \left.+\frac{d R_{3}(\theta)}{d \theta}\left(R_{2}(\theta)-R_{1}(\theta)\right)\left(R_{1}(\theta)-r^{\prime}\right)\right\}
\end{aligned}
$$

It is obvious that if the concentrator is circular, namely $d R_{1}(\theta) / d \theta=d R_{2}(\theta) / d \theta=d R_{3}(\theta) / d \theta=0$, then $\lambda$ vanishes.
We report in Fig. 2 some finite element results for a concentrator when we apply constant temperature value normalized to 1 and -1 respectively on the left side and right side for a steady state and without heat source. Without loss of generality, the irregular boundaries of the cloak are defined on some different contour equations: Case 1:

$\left\{\begin{array}{l}R_{1}(\theta)=0.6+0.1 \cos (\theta)+0.05 \sin (2 \theta)-0.1 \sin (3 \theta) \\ R_{3}(\theta)=1.2+0.1 \sin (\theta)+0.2 \sin (3 \theta)\end{array}\right.$

Case 2:

$R_{1}(\theta)=0.6$;

$R_{3}(\theta)=1.2+0.1 \sin (\theta)+0.2 \sin (3 \theta)$

Case 3:

$R_{1}(\theta)=0.6+0.1 \cos (\theta)+0.05 \sin (2 \theta)-0.1 \sin (3 \theta) ;$

$R_{3}(\theta)=1.2$

Case 4:

$R_{1}(\theta)=0.6 ; R_{3}(\theta)=1.2$

One should note that the boundary $R_{2}(\theta)$ is a virtual one and only serves the purpose of the geometric transformation. Therefore, we define a circular boundary with a radius of 0.85 for simplification, namely $R_{2}(\theta)=0.85$ in the cases.

Temperature field profiles for different cases are shown in Fig. 2. One can see that the isothermal lines within the domain $R_{1}(\theta) \leq r^{\prime} \leq R_{2}(\theta)$ are bent significantly towards the inner core, which increases the temperature gradient and thus the overall heat flow in the inner domain without affecting the exterior heat profile.

It is clear that if the concentrator is circular then the coefficient $\lambda$ vanishes. A perfect cylindrical thermal concentrator is thus achieved, as is shown in Fig. 2 (d). The concentrator compresses the heat flux of the annulus into the small inner domain such that the energy density in that region is considerably enhanced while remaining uniform. It is found that this particular concentrator increases the heat flux gradient by $42 \%$ in the region $r^{\prime} \leq R_{1}(\theta)$.

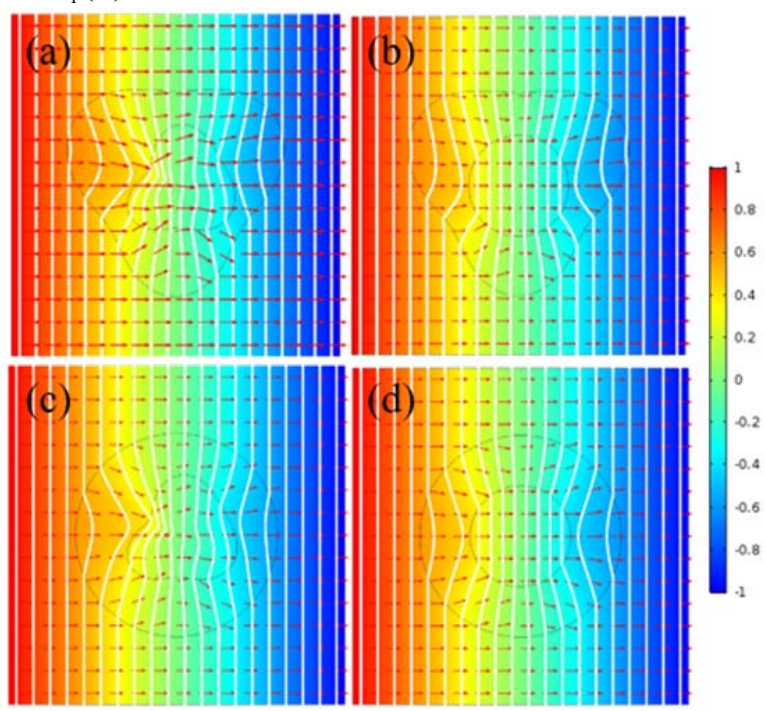

Fig. 2. Temperature field profiles for different cases of a concentrator: (a) Case 1; (b) Case 2; (c) Case 3; (d) Case 4. 


\section{Conclusions}

In summary, this paper studied analytically and numerically the extension of transformation optics technique to the application of thermodynamics. We proposed three-dimensional heat flux cloaks and concentrators with arbitrarily shaped non-conformal objects and coatings. Such a cloak reduced the temperature inside the object without interfering with outside thermal field while a concentrator would significantly increase the heat density in a tiny region.

It is stressed that the proposed method is capable of controlling the diffusive heat current, including cloaking a given region and focusing heat flows into a domain of interest. The method we propose will extend the inherent design flexibility in transformation thermodynamics.

\section{Acknowledgement}

This work was supported by the National Natural Science Foundation of China (Grant Nos. 11732002, 11672089 and 11421091), the Fundamental Research Funds for the Central Universities (Grant No. HIT.NSRIF.2017017), Natural Science Foundation of Heilongjiang Province, China (Grant No. A2017003).

\section{References}

1. J.B. Pendry, D. Schurig, D.R. Smith, Science 312, 1780 (2006)

2. U. Leonhardt, Science 312, 1777 (2006)

3. M. Farhat, S. Guenneau, S. Enoch, A. Movchan, F. Zolla, A. Nicolet, New J. Phys. 10, 115030 (2008).

4. S.A. Cummer, D. Schurig, New J. Phys. 9, 45 (2009).
5. A.N. Norris, Proc. R. Soc. A 464, 2411 (2008).

6. D. Schurig, J.J. Mock, B.J. Justice, S.A. Cummer, J.B. Pendry, A.F. Starr, D.R. Smith, Science 314, 977 (2006).

7. F. Zolla, S. Guenneau, A. Nicolet, J.B. Pendry, Opt. Lett. 32, 1069 (2007).

8. F. Magnus, B. Wood, J. Moore, K. Morrison, G. Perkins, J. Fyson, M. Wiltshire, D. Caplin, L. Cohen, J. B. Pendry, Nat. Mater. 7, 295 (2008).

9. M. Brun, S. Guenneau, A.B. Movchan, Appl. Phys. Lett. 94, 061903 (2009).

10. G.W. Milton, M. Briane, J.R. Willis, New J. Phys. 8, 248 (2008).

11. A. Greenleaf, Y. Kurylev, M. Lassas, G. Uhlmann, New J. Phys. 10, 115024 (2008).

12. S. Zhang, D.A. Genov, C. Sun, X. Zhang, Phys. Rev. Lett. 100, 123002 (2008).

13. C.Z. Fan, Y. Gao, J.P. Huang, Appl. Phys. Lett. 92, 251907 (2008).

14. X.Y. Shen, C.R. Jiang, Y. Li, J.P. Huang, Appl. Phys. Lett. 109, 201906 (2016).

15. T. Chen, C.N. Weng, J.S. Chen, Appl. Phys. Lett. 93, 114103 (2008).

16. S. Guenneau, C. Amra, D. Veynante, Opt. Express 20, 8207 (2012).

17. S. Narayana, Y. Sato, Phys. Rev. Lett. 108, 214303 (2012)

18. R. Schittny, M. Kadic, S. Guenneau, M. Wegener, Phys. Rev. Lett. 110, 195901 (2013).

19. T. Yang, L. Huang, F. Chen, W. Xu, J. Phys. D: Appl. Phys. 46, 305102 (2013). 